\title{
ORIGINAL ARTICLE \\ The efficiency of close inbreeding to reduce genetic adaptation to captivity
}

\begin{abstract}
K Theodorou ${ }^{1}$ and D Couvet ${ }^{2}$
Although ex situ conservation is indispensable for thousands of species, captive breeding is associated with negative genetic changes: loss of genetic variance and genetic adaptation to captivity that is deleterious in the wild. We used quantitative genetic individual-based simulations to model the effect of genetic management on the evolution of a quantitative trait and the associated fitness of wild-born individuals that are brought to captivity. We also examined the feasibility of the breeding strategies under a scenario of a large number of loci subject to deleterious mutations. We compared two breeding strategies: repeated half-sib mating and a method of minimizing mean coancestry (referred to as $\mathrm{gc} / \mathrm{mc}$ ). Our major finding was that halfsib mating is more effective in reducing genetic adaptation to captivity than the gc/mc method. Moreover, half-sib mating retains larger allelic and adaptive genetic variance. Relative to initial standing variation, the additive variance of the quantitative trait increased under half-sib mating during the sojourn in captivity. Although fragmentation into smaller populations improves the efficiency of the gc/mc method, half-sib mating still performs better in the scenarios tested. Half-sib mating shows two caveats that could mitigate its beneficial effects: low heterozygosity and high risk of extinction when populations are of low fecundity and size and one of the following conditions are met: (i) the strength of selection in captivity is comparable with that in the wild, (ii) deleterious mutations are numerous and only slightly deleterious. Experimental validation of half-sib mating is therefore needed for the advancement of captive breeding programs.
\end{abstract}

Heredity (2015) 114, 38-47; doi:10.1038/hdy.2014.63; published online 23 July 2014

\section{INTRODUCTION}

Captive breeding programs are necessary to avoid the extinction of many endangered species. They are commonly used for the conservation of species that are now extinct in the wild or as reinforcement to declining wild populations via recurrent translocations (Christie et al., 2012). It has been predicted that over 3000 vertebrate species and subspecies will require captive breeding programs to achieve their conservation (Frankham, 2008).

A central goal of ex situ conservation is to conserve the ability of captive populations to survive and reproduce when reintroduced to the wild environment. However, captive-born individuals usually have reduced fitness when reintroduced into the wild (Snyder et al., 1996; Araki et al., 2008; Christie et al., 2012), which results in a significantly lower reintroduction success of captive-born populations in comparison with translocations of wild-caught individuals (Williams and Hoffman, 2009).

Several adverse genetic changes may occur in captivity that could reduce the success rate of captive breeding programs: (i) inbreeding depression, (ii) accumulation of deleterious mutations, (iii) loss of genetic diversity and (iv) genetic adaptation to captivity (Snyder et al., 1996; Araki et al., 2008; Christie et al., 2012). These changes are associated with the usually small size of captive populations (high inbreeding and genetic drift), the differences between the captive and the wild environment (relaxed selection due to the benign captive environment and artificial or natural selection to captive conditions) or the interaction of these factors.

Effective management regimes have been recommended to address the issue of loss of genetic diversity, inbreeding depression and mutation accumulation (Allendorf, 1993; Ballou and Lacy, 1995; Wang, 2000; Fernández and Caballero, 2001a; Theodorou and Couvet, 2003; Fernández et al., 2004). Although there is mounting evidence that genetic adaptation to captivity occurs rapidly for many different organisms and can affect strongly the fitness of reintroduced individuals (Hedrick and Fredrickson, 2008; Jule et al., 2008), little work has been done on the development of breeding strategies aiming at minimizing genetic adaptation. Equalization of family sizes or breeding strategies of minimizing mean coancestry are proposed to counteract genetic adaptation (Williams and Hoffman, 2009). These strategies are recommended to achieve the other goals of genetic managements, that is, to lower the loss of genetic diversity and the effects of inbreeding depression (Fernández and Caballero, 2001a, b; Theodorou and Couvet, 2003; Meffert et al., 2005). Although equalization of family sizes is expected to halve the rate of genetic adaptation (Allendorf, 1993), experimental results raise doubts about the ability of such breeding schemes to reduce genetic adaptation to captivity and, thus, increase fitness on reintroduction (Loebel et al., 1992; Montgomery et al., 1997; Frankham et al., 2000; Wisely et al., 2003).

${ }^{1}$ Biodiversity Conservation Laboratory, Department of Environment, University of the Aegean, Mytilene, Greece and 2 UMR $7204 C E S C O$ MNHN-CNRS-UPMC, CP 51, Paris, France

Correspondence: Dr K Theodorou, Biodiversity Conservation Laboratory, Department of Environment, University of the Aegean, University Hill, 81100 Mytilene, Greece. E-mail: ktheo@aegean.gr

Received 22 October 2013; revised 2 April 2014; accepted 21 May 2014; published online 23 July 2014 
With this work, we propose a breeding strategy that effectively minimizes genetic adaptation to captivity: repeated half-sib matings (or synonymously circular mating) (Kimura and Crow, 1963). In a previous paper (Theodorou and Couvet, 2010), we showed that this breeding scheme achieves two primary goals of ex situ genetic management: (i) retains high levels of genetic diversity, and (ii) reduces the genetic load resulting, therefore, in increased viability of reintroduced populations.

To evaluate the performance of half-sib matings, we compared it with the $\mathrm{gc} / \mathrm{mc}$ method proposed by Fernández and Caballero (2001b) and an unmanaged randomly mating population used as control. We made comparisons with the $\mathrm{gc} / \mathrm{mc}$ method, because it has been found to be the most effective method in terms of genetic diversity and population fitness (see Ivy and Lacy (2013)).

We also assess how the different breeding strategies perform when the captive population is subdivided into several subpopulations. Fragmentation is expected to reduce genetic adaptation to captivity because the effect of selection decreases when the number of individuals decreases (Williams and Hoffman, 2009 and references therein). We explore, therefore, the result of the interaction between breeding strategies and fragmentation on genetic adaptation.

We used a quantitative genetic model to explore these questions. We studied how differences in the captive and the wild environment (both in the optimum phenotype and in the intensity of selection) shape the distribution of a quantitative trait and, thus, fitness on reintroduction in relation to the breeding strategy. If populations adapt to captive conditions, the distribution of the trait moves toward the optimum genotypic value in the captive environment, which is likely to be different than the optimum in the wild. The difference between the captive and wild optimum genotype, the relaxation of selection in captivity and the extent of the population's genetic adaptation to the captive environment will determine the decline in fitness when the captive-born individuals are reintroduced into the wild. The quantitative trait is determined by a finite number of freely recombining additive loci. As pointed out by Ford (2002), such an approach is expected to provide a better description of phenotypic evolution than single-locus models.

Repeated half-sib mating, as a form of close inbreeding, may reduce substantially fitness due to inbreeding depression (Caballero and Toro, 2002). It is, therefore, of great interest to investigate whether this reduction in fitness would be sustainable for the captive populations or whether inbreeding would mitigate the efficacy of half-sib mating by increasing the extinction probability of the captive populations to unacceptable levels. We, therefore, examine the feasibility of the breeding strategies under a scenario of a large number of loci subject to deleterious mutations. Moreover, by coupling the effect of adaptation to captivity and inbreeding depression, we permit a broader comparison of the different breeding strategies as conservation tools.

\section{MATERIALS AND METHODS}

Quantitative genetic model

The genetic assumptions of our model are mostly drawn from Burger et al. (1989); Burger and Lynch (1995) and Ford (2002).

We considered the evolution of a single trait and assumed that the genotypic value, $G$, of the trait is determined by $L=50$ freely recombining loci. We assumed that allelic effects on the trait are additive both within each locus and across loci. The phenotypic value of an individual, $Z$, is obtained by adding to its genotypic value a random environmental effect distributed normally with mean 0 and variance $\sigma_{\mathrm{e}}^{2}=1$ (Burger and Lynch, 1995).
We assumed that stabilizing selection is acting on the phenotypic trait. The fitness of an individual with phenotype $Z$ is assumed, therefore, to be

$$
W_{\mathrm{q}}(Z)=\exp \left[-\frac{\left(Z-\theta_{i}\right)^{2}}{2 \omega^{2}}\right]
$$

Where $\omega^{2}$ is inversely proportional to the strength of stabilizing selection and $\theta_{i}$ is the optimal phenotype in the environment $i$. The Gaussian fitness function seems an appropriate choice for many quantitative traits (Willensdorfer and Burger, 2003). Notice that due to the fitness function used, the effects of each allele are additive on the trait but they are not necessarily on fitness (Pavlidis et al., 2012), that is, alleles with effects far away from the optimum genotype will have a larger effect on fitness decline relatively to alleles with effects near the optimum.

The number of alleles segregating simultaneously at each locus is unlimited. Mutation creates a new allele with allelic effect drawn by a Gaussian distribution with mean zero and variance $\alpha^{2}$ with regard to the current allelic effect. Mutations occur independently at each locus on each gamete with probability $\mu$. The diploid genomic mutation rate is set to $U=2 L \mu=0.02$ and the variance of the allelic effect to $\alpha^{2}=0.05$. These values result to a mutational variance $\sigma_{\mu}^{2}=U a^{2}=10^{-3} \sigma_{\mathrm{e}}^{2}$ as suggested by the literature (Burger $e$ t al., 1989). We also ran simulations for lower mutation rates with larger effects on the trait (that is, higher $\alpha^{2}$ ) with no appreciable difference in our results.

In our model, the captive environment differs from the wild one in two aspects. First, fitness in the wild is maximized for individuals with trait value $Z=\theta_{\mathrm{w}}$, whereas the optimal trait value in captivity is $Z=\theta_{\mathrm{c}}$. Hence, there is a difference in the optimum phenotype between the two environments $\Delta \theta=$ $\left|\theta_{\mathrm{w}}-\theta_{\mathrm{c}}\right|$ (see Figure 1 in Ford (2002)). Second, selection in captivity is weaker compared with the wild. In our simulations, the strength of selection in the wild is set to $\omega_{w}^{2}=3$ as suggested by Estes and Arnold (2007). In captivity, we assumed that selection is 10 times weaker $\left(\omega_{\mathrm{c}}^{2}=30\right)$.

To assess the efficiency of the different breeding strategies to mitigate genetic adaptation to captive conditions, we used two measures: (i) the shift of the population mean genotypic value toward the optimum genotypic value in the captive environment and (ii) the fitness decline due to adaptation to captivity when population is reintroduced to the wild. We made the assumption that the captive program is initialized by individuals that originate from the wild. Hence, the trait distribution in the initial generation in captivity is at an optimum that has been shaped in the wild environment (that is, $M_{\mathrm{g}}(t=0)$ $=0)$. We set the difference in the optimum genotype between captivity and the wild, $\Delta \theta=5$, which corresponds to large differences between the two environments (five times the initial additive genetic variance). However, the relative response of the different breeding systems does not depend on $\Delta \theta$ (results not shown).

\section{Deleterious mutations}

The overall effect of deleterious mutations was considered in a previous work (Theodorou and Couvet, 2010. In this work, we ran a few simulations to examine in particular their effect on the sustainability of the breeding strategies under study (Table 1) and fitness on reintroduction.

We considered two different mutational models for deleterious alleles: (i) Model I, which assumes that deleterious mutations occur at high rate but are slightly deleterious (Mukai et al., 1972; Lynch et al., 1999) with mutational parameters $U_{\mathrm{d}}=1, \bar{s}=0.05, \bar{h}=0.35$, where $U_{\mathrm{d}}$ is the mutation rate per diploid genome and generation, $\bar{s}$ and $\bar{h}$ are respectively the mean selection and dominance coefficient and (ii) Model II according to which mutations are considerably less frequent but of larger effect (Caballero and Keightley, 1994; García-Dorado and Caballero, 2000) with mutational parameters $U_{\mathrm{d}}=0.06$, $\bar{s}=0.264, \bar{h}=0.2$. The selection coefficient at each locus was sampled from a Gamma distribution with shape parameter $\beta$ and mean $\bar{s}$. The shape parameter was set to $\beta=1$ and 2.3 for model I and II, respectively (de Cara et al., 2013). The dominance coefficient of each mutation was sampled from a uniform distribution in the range $\left[0, e^{-k s}\right]$, where $k$ is a constant that gives the required $\bar{h}$. We also considered a class of rare highly recessive lethal mutations with mutational parameters $U_{1}=0.03, s_{1}=1, h_{1}=0.02$. Fitness was controlled by 1000 freely recombining biallelic loci. The number of loci is large enough to allow mutations to accumulate without saturating the genome within the 
Table 1 Probability of extinction during captive breeding

25th generation

\begin{tabular}{|c|c|c|c|c|c|c|c|c|}
\hline \multirow{2}{*}{$N / F$} & \multicolumn{2}{|c|}{2.4} & \multicolumn{2}{|c|}{3.0} & \multicolumn{2}{|c|}{3.6} & \multicolumn{2}{|c|}{4.2} \\
\hline & Half-sib & $g c / m c$ & Half-sib & $g c / m c$ & Half-sib & $g c / m c$ & Half-sib & $g c / m c$ \\
\hline 20 & 66.0 & 10.2 & 5.0 & 0.4 & 0.4 & 0 & 0 & 0 \\
\hline 30 & 19.8 & 0.8 & 0 & 0 & 0 & 0 & 0 & 0 \\
\hline 40 & 4.4 & 0 & 0 & 0 & 0 & 0 & 0 & 0 \\
\hline 50 & 0.4 & 0 & 0 & 0 & 0 & 0 & 0 & 0 \\
\hline
\end{tabular}

50th generation

\begin{tabular}{|c|c|c|c|c|c|c|c|c|}
\hline \multirow[t]{2}{*}{$N / F$} & \multicolumn{2}{|c|}{2.4} & \multicolumn{2}{|c|}{3.0} & \multicolumn{2}{|c|}{3.6} & \multicolumn{2}{|c|}{4.2} \\
\hline & Half-sib & $g c / m c$ & Half-sib & $g c / m c$ & Half-sib & $g c / m c$ & Half-sib & $g c / m c$ \\
\hline 20 & 96.2 & 82.8 & 33.6 & 28.0 & 7.8 & 5.2 & 0.8 & 1.0 \\
\hline 30 & 62.8 & 19.6 & 1.2 & 0.6 & 0 & 0 & 0 & 0 \\
\hline 40 & 20.8 & 2.0 & 0 & 0 & 0 & 0 & 0 & 0 \\
\hline 50 & 4.0 & 0 & 0 & 0 & 0 & 0 & 0 & 0 \\
\hline
\end{tabular}

Deleterious mutations follow the mutational model I (see text). The coefficient of selection for deleterious alleles is set to $s_{i}^{\prime}=s_{i} / 2$. Parameters related to the quantitative trait subject to adaptation to captivity are as in Figure 2.

$N$ : population size; $F$ : number of progeny per mating.

number of generations considered. Loci act multiplicatively on fitness, that is, the genetic fitness of an individual is given by the relationship

$$
W_{\mathrm{m}}=W_{\mathrm{d}} W_{\mathrm{l}},
$$

Where

$$
\begin{aligned}
& W_{\mathrm{d}}=\prod_{i=1}^{n_{\text {het }}^{\mathrm{d}}}\left(1-h_{i} s_{i}\right) \prod_{i=1}^{n_{\text {hom }}^{\mathrm{d}}}\left(1-s_{i}\right) \\
& W_{\mathrm{l}}=\left(1-h_{1} s_{\mathrm{l}}\right)^{n_{\text {het }}^{i}}\left(1-s_{i}\right)^{n_{\text {hom }}^{i}}
\end{aligned}
$$

is respectively the fitness across loci for mildly deleterious and lethal mutations and $n_{\text {het }}^{\mathrm{d}, \mathrm{l}}\left(n_{\text {hom }}^{\mathrm{d}, \mathrm{l}}\right)$ is the number of heterozygous (homozygous) loci. We considered that selection against mildly deleterious mutations is in some extent relaxed in captivity but not for lethals (Ralls et al., 2000); we assumed that in captivity, $s_{i}^{\prime}$ ranges from $s_{i}^{\prime}=s_{i} / 2$ to $s_{i} / 10$ and $h_{i}^{\prime}$ is then found following the procedure described above.

Finally, the total fitness of an individual is given by the product of the fitness, which is due to the value of the quantitative trait, and the fitness due to deleterious mutations,

$$
W=W_{\mathrm{q}} W_{\mathrm{m}}
$$

Both the quantitative trait and deleterious mutations are assumed to affect fecundity and survival with effect $\sqrt{W}$ on each vital rate (Fernández and Caballero, 2001a).

\section{Initial conditions}

To initialize the population, $N$ genetically unrelated individuals are generated by choosing random allelic effects for each allele that controls the quantitative trait. We, then, normalize the allelic effects to obtain a population genetic variance with zero mean and unit standard deviation $N(0,1)$ (Bjorklund et al., 2009). The initial individuals are assumed to be adapted to the wild environment, that is, the mean genotypic value, $M_{g}$, coincides with the optimum phenotype in the wild $\left(\theta_{w}=0\right)$.

To determine the distribution of deleterious alleles among individuals and loci, we sampled randomly from an infinite population at mutation-selection balance (see Theodorou and Couvet, 2004 for more details).
We ran 500 replicates for each scenario, and we calculated the mean of each parameter of interest (fitness, mean genotypic value, genetic variance) in each generation of the captive program and at reintroduction.

All simulations were performed in Delphi 7 and the code is available on request.

\section{Genetic variance}

In each generation, we measured both the additive genetic variance of the quantitative character, $V_{\mathrm{g}}$, and the neutral genetic variation $(A$ : mean number of alleles/locus and $H$ : mean observed heterozygosity/locus) in the captive population. In the case of fragmented populations, genetic variation is calculated in the metapopulation level, that is, if all individuals were pooled together. In this way, we assess the genetic variation that a reintroduced population would have if it had been initialized by all the captive individuals.

To measure neutral genetic variation, we assumed 200 freely recombining neutral loci. At the initial generation, population has at each locus $2 \mathrm{~N}$ alleles (where $N$ denotes population size) and, therefore, heterozygosity equals 1 .

\section{Life cycle}

Our simulations consider a captive population that is founded by $N$ individuals from the wild. The initial population size, $N$, is equal to carrying capacity in captivity, that is, the populations may decline but they can never exceed $N$ (ceiling model); sex is randomly assigned to individuals. In each generation, the order of events was mutation, reproduction, viability selection and, for the fragmented population scenarios, migration. Generations are discrete and non-overlapping.

The choice of breeding pairs and the production of offspring differ according to the breeding strategy. We simulated three breeding strategies:

(1) The Gc/mc method. A management procedure that seeks to minimize group coancestry proposed by Fernández and Caballero (2001b). To simulate this method, we followed the description of Fernández and Caballero (2001b) and references therein. This method comprises two steps. First, the contribution of each parent in the next generation was chosen in such a way that group coancestry of the offspring was minimized. The optimization was performed following the procedure 
'simulated annealing' as described in Fernández and Toro (1999). Once the contribution of each parent was determined, we chose the combination of matings that yielded the lowest average pairwise coancestry between couples. The minimization was performed using the 'Hungarian algorithm' (Kuhn, 1955). To simulate departures from the predefined schemes, as could be the case in real situations, we proceeded as in Theodorou and Couvet (2010), that is, each mating has $F_{f m}(t)$ chances to produce a viable offspring, where $F_{f m}(t)=\sqrt{F_{f}(t) F_{m}(t)}$ is the expected fecundity per couple; $F_{i}=F_{\max } \sqrt{W_{i}}, i=f, m$ is the fecundity of the father and the mother, respectively, and $F_{\max }$ is a scaling factor to obtain the desired initial number of progeny. The viability of the $F_{f m}(t)$ offspring was given by Equation (1) or (3) when deleterious mutations were considered-and compared with a uniformly distributed random number in the range $[0,1]$ to decide whether a viable offspring was produced. One of the viable offspring (if any) was used to form the next generation; the other viable offspring formed the stock pool so as they can be used subsequently. The procedure was continued until all couples reproduced. If a couple did not produce any viable offspring, then we chose a viable one from the other couples (that is, the stock pool). We chose those individuals from the stock pool that confer to the population the minimum mean pairwise coancestry, in accordance to the goal of the $\mathrm{Gc} / \mathrm{mc}$ method. If no extra viable offspring was produced then the population declines until extinction eventually occurs.

(2) Repeated half-sib mating proposed by Theodorou and Couvet (2010). For this method, matings were realized according to Figure 1. Viability selection was simulated as for the $\mathrm{Gc} / \mathrm{mc}$ method. When a failure occurred, that is, a couple did not reproduce any viable offspring, we chose an individual from the stock pool that shared one of the parents with the 'childless' couple. If such an offspring did not exist, we then chose one at random from the stock pool. Once again, if the individuals in the stock cannot replace the number of failures, the population size decreases until extinction eventually occurs.

(3) a random mating population that serves as a reference case. According to this mating system, individuals are drawn at random to form the N/2 breeding pairs. Viability selection and offspring production is identical to that described for the $\mathrm{Gc} / \mathrm{mc}$ procedure.

\section{Population fragmentation}

We considered scenarios where the captive population is divided into several smaller populations. We considered two cases: (i) populations are completely isolated during the captive program, and (ii) each population exchanges one migrant every generation with the other populations; the migrants, as well as the population where the migrant goes, are chosen at random. Thus, the number of immigrants equals the number of emigrants. As it was shown by Bouchy et al. (2005), this is a migration pattern that retains high levels of genetic variation at the metapopulation scale.

\section{RESULTS}

\section{Genetic adaptation to captivity}

It is clear from our results that repeated crosses between half-sibs lead to the lowest genetic adaptation to captivity. In relation to the $\mathrm{gc} / \mathrm{mc}$ method, the shift in the trait distribution after 50 generations in captivity under half-sib mating is $35-67 \%$ slower for population sizes of 20-100 individuals, respectively (Figure 2). For larger populations, we observe a higher rate of adaptation to captivity under random mating or the $\mathrm{gc} / \mathrm{mc}$ method; the more effective selection associated with larger populations sizes drive faster the trait distribution toward the new equilibrium. In contrast, the rate of trait shift under half-sib matings does not depend on population size.

Another way to measure the extent of adaptation to captive conditions is the fitness decline on reintroduction. Variation in fitness according to the breeding strategy yields the same results as the mean genotypic value, that is, half-sib mating maximizes population fitness on reintroduction due to the weaker adaptation to captivity (Figure 3).

To distinguish which part of fitness decline results solely from relaxation of selection due to the benign captive conditions and which is due to both relaxation and adaptation, we ran simulations with $\Delta \theta=0$, that is, the difference between the captive and the wild selection is only in the strength of selection (which is determined by $\omega^{2}$ ) and not in the optimum genotype. According to Figure 3, relaxed selection contributes moderately in fitness reduction with all the breeding systems having similar responses to selection relaxation. In contrast, adaptation to captivity can lead to severe fitness reduction that could jeopardize the viability of reintroduced population. Halfsib mating can, however, attenuate the negative consequences of adaptation to captivity. For the parameter values used in our analysis, fitness reduction is lower by $25-57 \%$ (for $N=20$ and 100 , respectively) under half-sib mating than under the $\mathrm{gc} / \mathrm{mc}$ method.

\section{Population fragmentation}

Population fragmentation is proposed as an efficient method to minimize genetic adaptation to captivity. We ran, therefore, some simulations where the founding population is subdivided into several subpopulations. Figure 4, shows the decline in fitness at reintroduction after a 50-generation captive program in the case of (i) complete isolation $(\mathrm{Nm}=0)$ and (ii) populations exchanging one migrant per generation $(\mathrm{Nm}=1)$.

Complete isolation into several subpopulations has indeed a positive effect on the reintroduced population's fitness for both random mating and the $\mathrm{gc} / \mathrm{mc}$ method. In contrast, fitness for

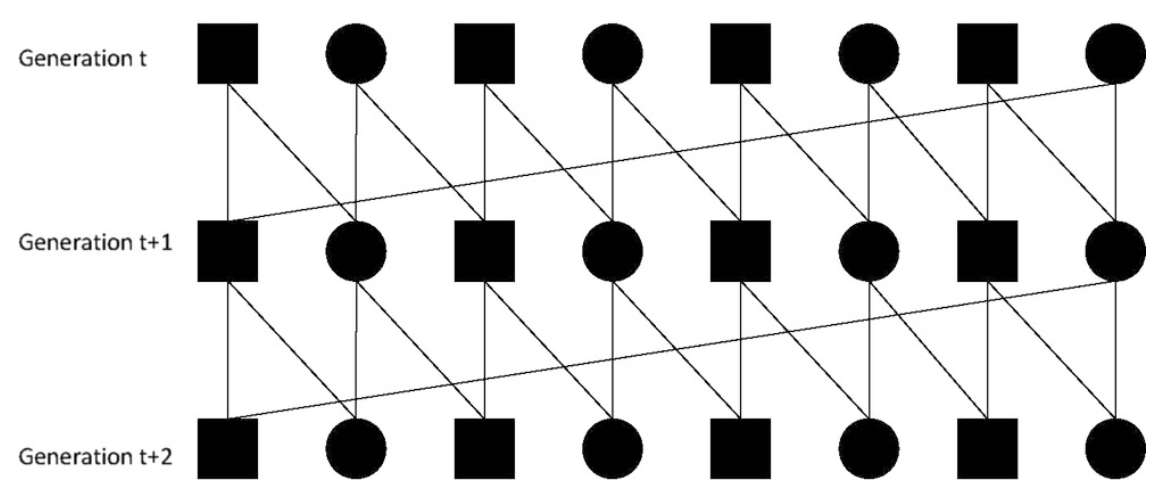

Figure 1 Schematic representation of three generations of individuals related with two sets of half-sib mating, for a population of eight individuals (four males, represented as squares, and four females, represented as circles). 


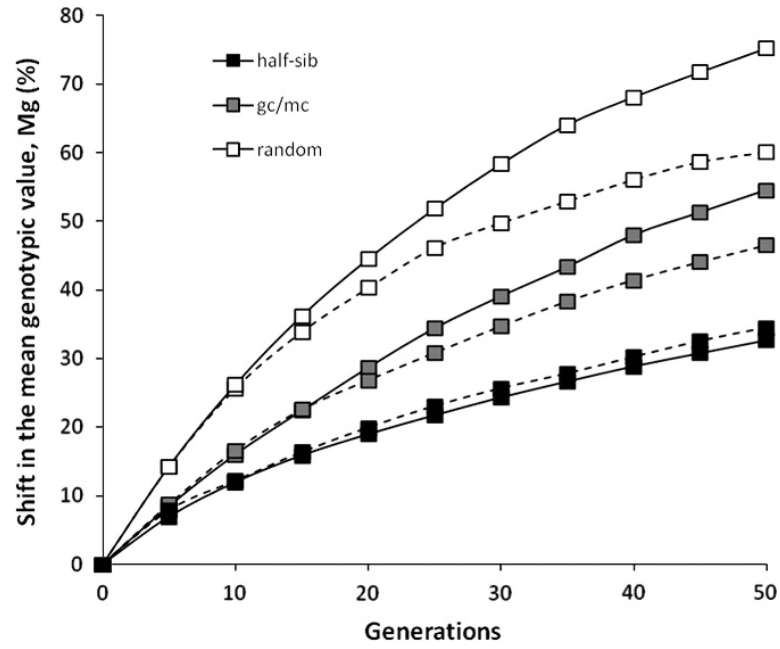

Figure 2 Rate of shift in the mean genotypic value, toward the captive optimum genotype. It is measured as the \% distance covered by the mean trait on the way to the new equilibrium for two population sizes: (i) $N=100$ (solid lines), and (ii) $N=20$ (dashed lines). The initial mean genotypic value of the population is set to $M_{\mathrm{g}}=0$, which coincides with the optimum genotypic value in the wild, $\theta_{w}$. The optimum genotypic value in captivity is set to $\theta_{c}=5$. Hence, a shift of $50 \%$ means that the mean genotypic value is half-way between the wild and the captive optimum genotypic value (that is, $M_{\mathrm{g}}=2.5$ ). The genotypic variance in the initial generation is 1 and the strength of selection is $\omega_{w}^{2}=3$, and $\omega_{c}^{2}=30$ for the wild and the captive environment, respectively. The number of progeny is set to four offspring/ mating. $M_{\mathrm{g}}$ represents the mean of 500 replicates; the variance was low and thus omitted from the figure. The same applies for the other results.

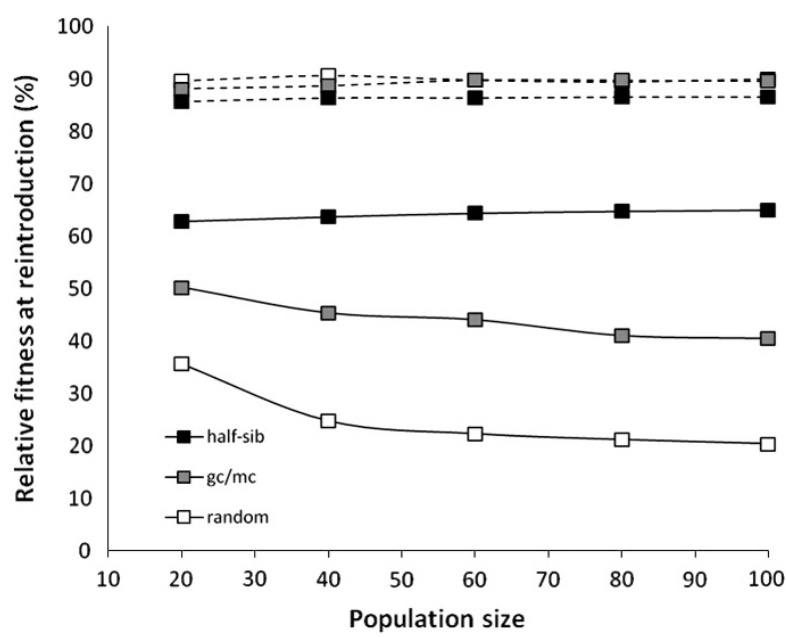

Figure 3 Relative fitness reintroduced to the wild populations after a 50generation captive program. Fitness is measured as the \% of fitness of the captive population relative to the fitness that the same population would have if it were kept to 'wild' conditions for the same period of time. We simulated two different captive environments, (i) $\theta_{\mathrm{c}}=\theta_{\mathrm{w}}=0$ (dashed lines), that is, the only difference between the wild and captivity is in the strength of selection and not in the optimum genotype, and (ii) $\theta_{c}=5$ (solid lines), as in Figure 2. Other parameter values are also as in Figure 2.

populations under half-sib mating is insensitive to fragmentation. When subpopulations are interconnected, fitness is slightly changed for random mating or the $\mathrm{gc} / \mathrm{mc}$ method, whereas it decreases under half-sib mating.

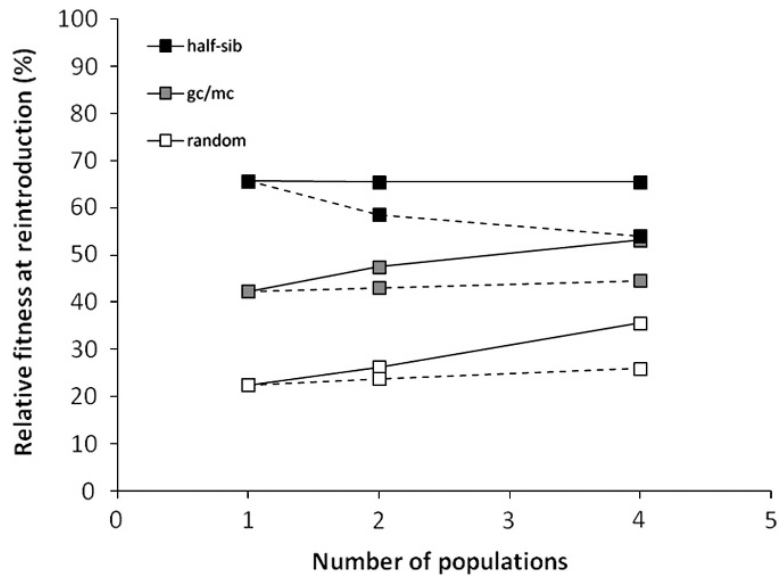

Figure 4 Relative fitness at reintroduction in the case of fragmented captive populations. Fitness is drawn against the number of distinct populations and is measured as in Figure 3. The total population size is set to $N=80$. We ran two fragmentation scenarios with regard to connectivity between populations: (i) populations are completely isolated ( $N m=0$; solid lines), (ii) populations exchange one migrant per generation $(\mathrm{Nm}=1$; dashed lines). Other parameter values are as in Figure 2.

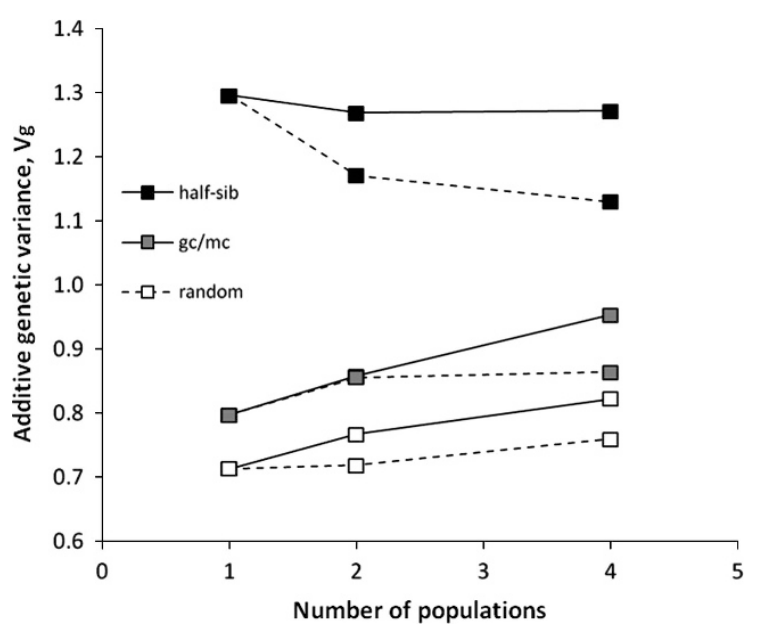

Figure 5 Additive genetic variance at the end of a 50 generations captive program for $N m=0$ (solid lines) and $N m=1$ (dashed lines). Parameter values are as in Figure 4.

Overall, although in the case of fragmented populations complete isolation reduces substantially genetic adaptation for random mating and the $\mathrm{gc} / \mathrm{mc}$ method, fitness at reintroduction would still be higher under half-sib mating. Connecting subpopulations via migration has small effects (for the $\mathrm{gc} / \mathrm{mc}$ method and random mating) or negative effects (for half-sib mating) on fitness at reintroduction.

\section{Genetic variation}

Additive genetic variance is maximized under half-sib mating. When this breeding strategy is combined with fragmentation, the genetic variance is reduced (when subpopulations are not completely isolated). Even if one takes into account this reduction, the genetic variance under half-sib mating remains considerably higher with regard to the genetic variance observed under the $\mathrm{gc} / \mathrm{mc}$ method (Figure 5). 


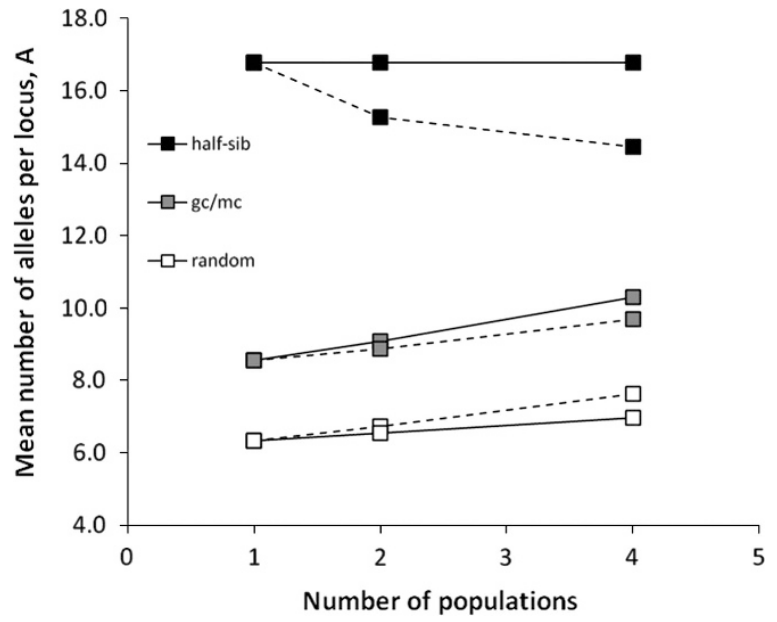

Figure 6 Mean number of alleles per locus at the end of a 50 generations captive program for $\mathrm{Nm}=0$ (solid lines) and $\mathrm{Nm}=1$ (dashed lines). Parameter values are as in Figure 4.

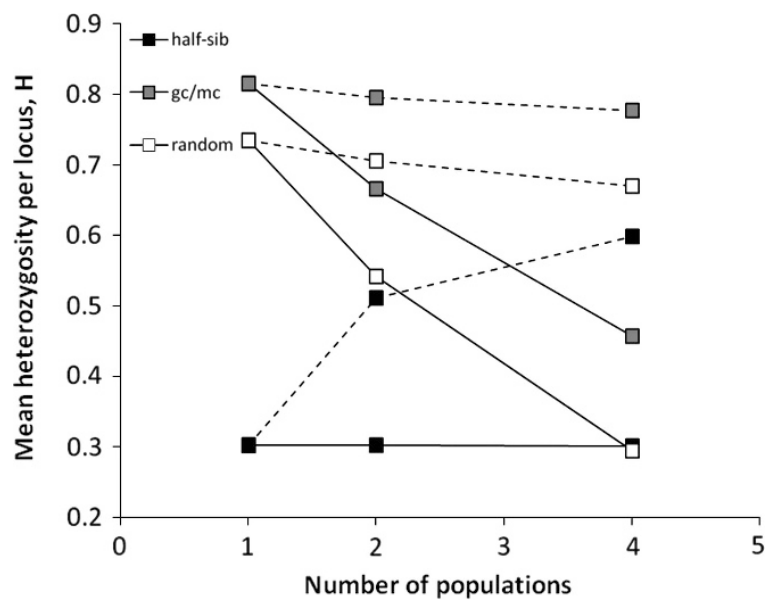

Figure 7 Mean heterozygosity per locus at the end of a 50 generations captive program for $\mathrm{Nm}=0$ (solid lines) and $\mathrm{Nm}=1$ (dashed lines). Parameter values are as in Figure 4.

Neutral variation is measured using the mean number of alleles and mean observed heterozygosity per neutral locus. The mean number of alleles follows closely the pattern observed for the additive genetic variance (Figure 6). This result indicates that the number of alleles is a better indicator of quantitative genetic variation than observed heterozygosity (compare Figures 6 and 7).

Very low values of observed heterozygosity are observed under halfsib mating. Such a low level of heterozygosity is typically associated with significant loss in fitness in experimental and zoo populations. Fragmentation followed by migration can remediate to some extent the loss of heterozygosity (Figure 7). In contrast, fragmentation, especially without migration, decrease heterozygosity under both random mating and the $\mathrm{gc} / \mathrm{mc}$ method.

\section{Joint effect of adaption to captivity and deleterious mutations}

To check the impact of inbreeding depression on the feasibility of half-sib mating and the $\mathrm{gc} / \mathrm{mc}$ method, we calculated the probability of extinction of the conserved population after 25 and 50 generations in captivity (Table 1 ).

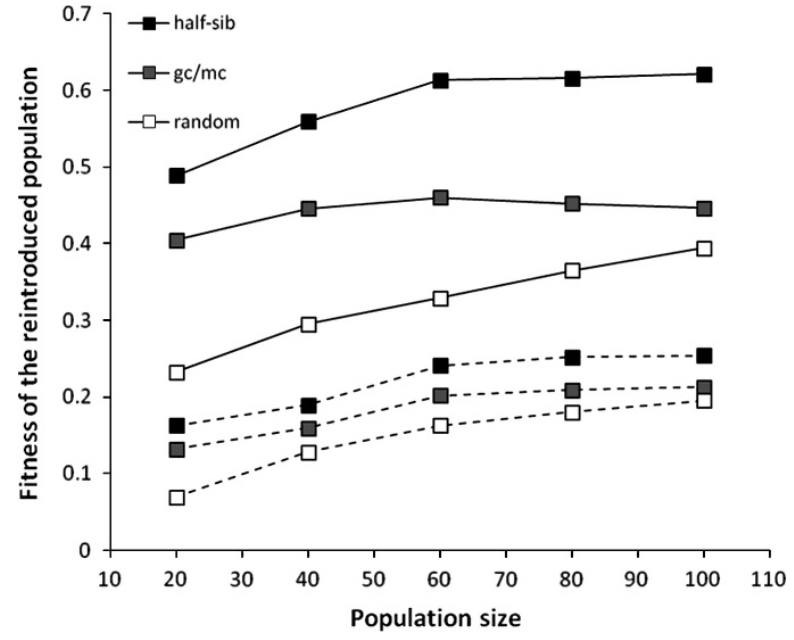

Figure $\mathbf{8}$ The joint effect of deleterious mutations and adaptation to captivity on mean population fitness in the first generation born under random mating in the wild after reintroduction. Mildly deleterious mutations follow the mutational model I (numerous slightly deleterious mutations). Two levels of selection relaxation are considered: (i) $s_{i}^{\prime}=s_{i} / 2$ (solid lines), (ii) $s_{i}^{\prime}=s_{i} / 10$ (dashed lines). Other parameter values are as in Figure 2.

Our results depend strongly on the mutational model considered. If we follow the view of less frequent but more detrimental mutations (Model II), inbreeding depression is not of a concern for neither of the breeding strategies. Probability of extinction is null for population sizes as small as 20 individuals and expected number of progeny of 2.2 offspring/mating (results not shown).

In contrast, if we consider numerous mutations of small effect (Model I), half-sib mating would lead to a high extinction risk for populations of low fecundity when population size is small.

To be sure, however, that we will avoid the negative effects of inbreeding depression, half-sib mating should be implemented only when the expected number of progeny is more than three offspring per mating and the population size does not fall under 30 individuals.

The combined effect of adaptation to captivity and inbreeding depression was assessed by calculating the mean population fitness in the first generation born under random mating in the wild after reintroduction (Figure 8). The relationship between population size and fitness is shaped by two antagonistic mechanisms: the effect of deleterious mutations, which decreases for larger populations and the effect of adaptation in captivity, which increases with population size (except for half-sib mating where it remains nearly constant).

The reintroduced population shows an important increase in fitness under half-sib mating when selection in captivity is highly relaxed. Differences between the two breeding strategies are somewhat decreased when selection in captivity is stronger. The reason is that stronger selection leads to a higher offspring mortality under half-sib mating and, thus, to a larger proportion of departures from the predefined scheme.

\section{DISCUSSION}

In this work, we addressed a central issue in ex situ conservation: which breeding strategy is the most effective in minimizing genetic adaptation to captivity. Genetic adaptation to captivity has been shown to be one of the most important mechanisms for the genetic deterioration of reproductive fitness of captive individuals when translocated to the wild (Frankham, 2008; Williams and Hoffman, 2009). An effective genetic management that minimizes genetic 
adaptation to captivity should, therefore, increase the success rate of reintroduction programs, which is the final goal of any ex situ conservation program.

\section{Advantages and drawbacks of half-sib mating}

Our major finding was that repeated half-sib mating is more effective in reducing genetic adaptation to the captive environment than methods of minimization of mean coancestry (for example, the $\mathrm{gc} / \mathrm{mc}$ method). When initiating an ex situ program by individuals born in the wild, half-sib mating lowers the shift of the quantitative trait toward the optimum captive genotype more effectively. Hence, individuals will have higher chances of survival and reproduction when introduced back to the wild. Interestingly, under half-sib mating the reduction of both genetic adaptation and fitness on reintroduction is equally if not more efficient in larger populations. This is in contrast with findings concerning the equalization of family size where adaptation is more of a concern in larger populations due to more effective selection (Woodworth et al., 2002).

Moreover, half-sib mating retains larger quantitative variation and allelic diversity than methods of minimization of mean coancestry. Relative to initial standing variation, the additive variance of the quantitative trait increased under half-sib matings during the sojourn in captivity. Evolutionary adaptation to the wild environment can only occur if the reintroduced population harbors sufficient genetic variation for selection to operate on (Hedrick, 2001). Thus, half-sib mating should confer higher viability to reintroduced populations both in the short-term, due to the lower rate of genetic adaptation to captivity, and in the long-term, due to the higher amount of quantitative genetic variance preserved during captive breeding.

Neutral variation is used as a proxy of adaptive variation. Two measures of neutral variation are usually employed: heterozygosity, which is supposed to predict the short-term selection response and number of alleles, which predicts the long-term evolutionary potential (Allendorf and Luikart, 2007, p.111). The maintenance of higher quantitative and allelic diversity under half-sib mating is important, as the optimal trait value in the wild could change during the sojourn of the population in captivity due to diverse causes (for example, climate change, habitat degradation). As the direction of such changes would be hard to predict, the population adaptive potential would depend strongly on the amount of genetic variation for selective traits but also on currently neutral variation, which is a potential target of selection in case of environmental change (Frankham, 1999).

Our results show that allelic diversity is very closely correlated with the additive variance and can be used as an indicator of the evolutionary potential retained by the different breeding strategies and population structures. The term heterozygosity is used, in our manuscript, for observed heterozygosity. The expected heterozygosity can be inferred by the number of alleles. Thus, half-sib mating has two opposing effects: it decreases the observed heterozygosity while increasing the expected heterozygosity. The important decrease in observed heterozygosity under half-sib mating raises concerns about the applicability of the method, as captive populations with such a low heterozygosity are shown to be at risk of extinction. The fact that under several scenarios, the risk of extinction under half-sib mating remains within the acceptable levels may be due to the fact that the decrease in heterozygosity is mainly due to intentional inbreeding and not due to genetic drift, which is known to have lower effects on fitness for the same level of inbreeding (Glémin, 2003). In any case, further investigation on the relation between low heterozygosity under half-sib mating and fitness loss is needed.
Two main reasons can explain why repeated half-sib mating lowers adaptation to captivity and retain higher quantitative variation. First, this breeding strategy eliminates the reproductive variance between families so that selection is limited to selection within families; in this aspect, half-sib mating is equivalent to equalization of family sizes. Second, different alleles tend to be fixed in the small, partially isolated sublines of closely related individuals, and this brings about a much slower ultimate rate of convergence to genetic homogeneity than in the case of minimization of mean coancestry (Nagylaki, 1992). For the same reason, half-sib mating retains more genetic variation than the $\mathrm{gc} / \mathrm{mc}$ method: although, in the short-term mating between relatives increase the average inbreeding in the population, forcing inbred matings results, in the long term, in a decrease in the amount of drift and, therefore, in higher genetic variance at the population level (Caballero and Toro, 2002).

Both methods perform better than random mating. Random mating involves random contributions from parents to progeny, and this is the main difference from $\mathrm{gc} / \mathrm{mc}$ and half-sib mating. Therefore, the comparison with $\mathrm{gc} / \mathrm{mc}$ and half-sib is on two aspects: the variation in contributions and the system of mating. Both aspects are responsible for the lower performance of random mating with regard to genetic adaptation and variance.

Nevertheless, half-sib mating is a form of intentional inbreeding, which is thought to have negative consequences on the viability of endangered species. The main criticism of intentional inbreeding is focused on issues related to: (i) purging efficiency; intentional inbreeding does not effectively purge deleterious alleles, because it reduces the effective population size, (ii) reduction of genetic variability and fixation of deleterious alleles; intentional inbreeding, with its associated reduction of the effective population size, could lead to the loss of adaptive variation and fixation of detrimental alleles (Keller et al., 2012). Although close inbreeding of related individuals causes a much more rapid initial decrease of heterozygosity than for minimization of mean coancestry, half-sib mating confers a variance-effective population size that is proportional to the square of population size (Crow and Kimura, 1970; Nagylaki, 1992). As a result, half-sib mating retains a substantially higher allelic variation, which is of great importance for the adaptation of the reintroduced populations. Moreover, (Theodorou and Couvet (2010)) showed that purging should be significantly more efficient under half-sib mating than under minimization of mean coancestry.

However, the high rate of inbreeding associated to half-sib mating could exacerbate the consequences of inbreeding depression jeopardizing, therefore, the applicability of this breeding scheme. Our analysis reveals that the effect of inbreeding depression on the feasibility of half-sib mating depends largely on (i) the level of selection relaxation in captivity; half-sib mating may fail to produce sustainable populations only when selection is comparable to that in the wild, and (ii) the distribution of deleterious mutations considered. If mutations are few and of large effect, then the impact of inbreeding depression is of lesser importance than if there are many slightly deleterious mutations. In the latter scenario, half-sib mating would indeed lead to a higher extinction risk for populations of low reproductive output in particular, if population is small. As a rough rule of thumb, half-sib mating should not be applied to populations with less than three offspring per brood. As for population size, it should exceed 30 individuals unless the species reproductive output is large (more than four offspring per brood). Thus, although there is still controversy about the most suitable mutational model, caution should be paid before implementing procedures that include close 
inbreeding to species of low rate of increase such as large mammals as they can impair population viability.

Two additional difficulties might arise from genetic management procedures. First, as both the $\mathrm{gc} / \mathrm{mc}$ method and circular mating require specific matings, they could be difficult to implement in social species, particularly where there are pair bonds and dominance hierarchies (Senner, 1980). Second, half-sib mating requires crosses between individuals that share the same mother, which can cause sexual inhibition (Ralls et al., 1988; Pusey and Wolf, 1996). Artificial insemination, which could resolve this problem, may lead to the loss of social-sexual behaviors. Although recent studies show that inbreeding avoidance is a subject of debate with some animals showing no avoidance or even preference to mate with relatives (Kokko and Ots, 2006; Jamieson et al., 2009), the majority of studies show that inbreeding is usually avoided. In a recent review article, Szulkin et al. (2013) point out that current theory is inefficient to understand inbreeding strategy. Hence, we can anticipate neither the difficulty to enforce half-sib mating nor the consequences associated with this breeding strategy for a given species.

Overall, to be cautious, half-sib mating should be applied to organisms that show relatively high reproductive rates and/or are unlikely to have social constraints when pairing and re-pairing individuals.

\section{Joint effects of fragmentation and breeding strategy on genetic adaptation}

To minimize genetic adaptation to captivity, the fragmentation of captive individuals to several smaller populations is recommended. This is a similar approach to half-sib mating, which subdivides the population into smaller units. With fragmentation, we seek to reduce the effective size within each subpopulation (or subline) while increasing the effective size of the whole population. Although fragmentation improves the efficiency of minimization of mean coancestry, half-sib mating still performs better, with regard to genetic adaptation and variance, in all the scenarios tested. Fragmentation has nearly no effect on the performance of half-sib mating. One has to choose whether to apply a within-population fragmentation via halfsib mating or a between-population fragmentation.

The two approaches (fragmentation and half-sib mating) share also a common drawback: the high rate of inbreeding (see the low heterozygosity associated with half-sib mating). High inbreeding rates can be associated with severe inbreeding depression. Inbreeding depression can impair the immediate population survival when its reproductive potential is low, which is usually the case for endangered species (O'Grady et al., 2006). However, if inbreeding becomes a concern, a small amount of gene flow between populations may be sufficient to reduce the rate of inbreeding (Backus et al., 1995; Fernandez et al., 2008). We applied a gene flow of one migrant per generation to assess the impact of migration on genetic adaptation and rate of inbreeding.

Migration cancels out the effect of fragmentation on genetic adaptation for the $\mathrm{gc} / \mathrm{mc}$ method, whereas it worsens the effect of half-sib mating. However, if we pair half-sib mating with low gene flow, we can achieve lower genetic adaptation than for the $\mathrm{gc} / \mathrm{mc}$ method and increase significantly the frequency of heterozygotes. It would be of interest to examine to what extent more elaborated patterns of exchanging individuals between populations would produce better results (Princée, 1995; Fernandez et al., 2008).

\section{Limitations of our approach}

Our approach comprises some simplifications with regard to both the genetic assumptions and the demography of the simulated populations. In the following, we point out some of these simplifications.

First, we simulated a single quantitative trait as being under adaptation to captivity. In real populations, selection in captivity may act on multiple traits (Araki et al., 2008) some of which may covary. To capture trait evolution in such a situation, the variancecovariance matrix, G, should be used (Lande and Arnold, 1983). Theory predicts that the population will evolve in the direction in which the population has the largest amount of covariation (see figure 1 in Hellmann and Pineda-Krch (2007)). Although, our results would certainly change quantitatively, this should not alter the relative performance of the mating systems; half-sib mating should continue to slow down adaptation due to higher genetic differentiation and absence of selection between the partially isolated mating units.

Second, although we considered that loci act independently, evidence shows a wide range of epistatic interactions (Roze and Blanckaert, 2014). Inbred mating systems, as half-sib mating, is expected to restrict the effective amount of recombination and, therefore, to facilitate the development of co-adapted gene complexes (Fenster et al., 1997). The enhancement of epistasis with inbreeding could have opposite effects whether interactions among loci tend to reinforce (negative epistasis) or compensate (positive epistasis) their deleterious effect (Kimura and Maruyama, 1966). The overall outcome should depend on the relative importance of negative and positive epistasis, which may vary with the magnitude of deleterious effects (Beerenwinkel et al., 2007) Further simulations are needed to explore this question.

Third, founder individuals are assumed to be genetically unrelated, which is rarely met in ex situ programs. However, the effects of halfsib mating, which are due to within-population subdivision and the absence of reproductive variance between families, should still hold under a variety of distributions of relatedness among initial individuals.

Fourth, our simulations consider discrete non-overlapping generations, which is not representative of most species subject to captive breeding. The relative performance of the different breeding strategies may change according to the species' life cycle. Ivy and Lacy (2013) showed that in the case of overlapping generations, the $\mathrm{gc} / \mathrm{mc}$ is outperformed, in terms of heterozygosity, by the procedure of minimization of mean kinship originally proposed by Ballou and Lacy (1995). In the case of half-sib mating, our results could be seen as a first approximation of matings between half-sibs that belong to the same cohort. However, it would be of importance to investigate in detail how it could be implemented and its performance in the case of more realistic life cycles, such as overlapping generations.

\section{CONCLUSION}

Overall, our results suggest that half-sib mating reduces the rate of genetic adaptation to captivity and retains high levels of adaptive genetic variance. Moreover, in a previous work, we showed that halfsib mating decreases the genetic load of captive-reared individuals and is robust to departures from the predefined breeding scheme (Theodorou and Couvet, 2010).

The genetic goals of ex situ management could also be achieved by the use of other systems of inbreeding than half-sib mating. Numerous such systems can be conceived (see Boucher and Cotterman, 1990). For instance, repeated first cousin mating is quite similar to half-sib mating and should show equivalent results with regard to adaptation and genetic variance. Compared with half-sib 
mating, first cousin mating should, in some extent, increase heterozygosity and decrease the rate of genetic uniformity (Campbell, 1991) while decreasing purging efficiency. Further comparison is needed to explore the robustness and effectiveness of different inbred mating systems.

In light of these results, we believe that experimental validation of half-sib mating - using for instance Drosophila species as model organism - would be of great interest for the advancement of captive breeding programs, to examine in particular if adapting the structural constraint of half-sib mating to the biology of the species concerned is necessary.

\section{DATA ARCHIVING}

Data available from the Dryad Digital Repository: doi:10.5061/ dryad.j51h4.

\section{CONFLICT OF INTEREST}

The authors declare no conflict of interest.

\section{ACKNOWLEDGEMENTS}

We thank the editor and the three referees for their valuable suggestions on the manuscript.

Allendorf FW (1993). Delay of adaptation to captive breeding by equalizing family-size. Conserv Biol 7: 416-419.

Allendorf FW, Luikart G (2007). Conservation and the Genetics of Populations. Blackwell: Malden, Massachusetts.

Araki $\mathrm{H}$, Berejikian BA, Ford MJ, Blouin MS (2008). Fitness of hatchery-reared salmonids in the wild. Evol App/ 1: 342-355.

Backus VL, Bryant EH, Hughes CR, Meffert LM (1995). Effect of migration or inbreeding followed by selection on low-founder-number populations-implications for captive breeding programs. Conserv Biol 9: 1216-1224.

Ballou JD, Lacy LC (1995). Identifying genetically important individuals for management of genetic diversity in pedigreed populations. In: Ballou JD, Gilpin M, Foose T (eds) Population Management for Survival and Recovery: Analytical Methods and Strategies in Small Population Conservation. Columbia university Press: New York, pp 76-111.

Beerenwinkel N, Pachter L, Sturmfels B, Elena SF, Lenski RE (2007). Analysis of epistatic interactions and fitness landscapes using a new geometric approach. BMC Evol Biol 7: 12.

Bjorklund M, Ranta E, Kaitala V, Bach LA, Lundberg P, Stenseth NC (2009). Quantitative trait evolution and environmental change. PLOS ONE 4: 2

Boucher W, Cotterman CW (1990). On the classification of regular systems of inbreeding. J Math Biol 28: 293-305.

Bouchy P, Theodorou K, Couvet D (2005). Metapopulation viability: influence of migration. Conserv Genet 6: 75-85

Burger R, Lynch M (1995). Evolution and extinction in a changing environment-a quantitative-genetic analysis. Evolution 49: 151-163.

Burger R, Wagner GP, Stettinger F (1989). How much heritable variation can be maintained in finite populations by mutation selection balance. Evolution 43: 1748-1766.

Caballero A, Keightley PD (1994). A pleiotropic nonadditive model of variation in quantitative traits. Genetics 138: 883-900.

Caballero A, Toro MA (2002). Analysis of genetic diversity for the management of conserved subdivided populations. Conserv Genet 3: 289-299.

Campbell RB (1991). On the robustness of regular systems of inbreeding. Math Biosci 104: $1-19$

Christie MR, Marine ML, French RA, Blouin MS (2012). Genetic adaptation to captivity can occur in a single generation. Proc Natl Acad Sci USA 109: 238-242.

Crow JF, Kimura M (1970). An Introduction to Population Genetics Theory. Harper \& Row: New York, USA.

de Cara MAR, Villanueva B, Toro MA, Fernandez J (2013). Purging deleterious mutations in conservation programmes: combining optimal contributions with inbred matings. Heredity 110: 530-537.

Estes S, Arnold SJ (2007). Resolving the paradox of stasis: Models with stabilizing selection explain evolutionary divergence on all timescales. Am Nat 169: 227-244.

Fenster CB, Galloway LF, Chao L (1997). Epistasis and its consequences for the evolution of natural populations. Trends Ecol Evol 12: 282-286.

Fernandez J, Toro MA, Caballero A (2008). Management of subdivided population in conservation programs: development of a novel dynamic system. Genetics 179 683-692.
Fernández B, Garcia-Dorado A, Caballero A (2004). Analysis of the estimators of the average coefficient of dominance of deleterious mutations. Genetics 168 1053-1069.

Fernández J, Caballero A (2001a). Accumulation of deleterious mutations and equalization of parental contributions in the conservation of genetic resources. Heredity $\mathbf{8 6}$ 480-488.

Fernández J, Caballero A (2001b). A comparison of management strategies for conservation with regard to population fitness. Conserv Genet 2: 121-131.

Fernández J, Toro MA (1999). The use of mathematical programming to control inbreeding in selection schemes. J Anim Breed Genet 116: 447-466.

Ford MJ (2002). Selection in captivity during supportive breeding may reduce fitness in the wild. Conserv Biol 16: 815-825.

Frankham R (1999). Quantitative genetics in conservation biology. Genet Res 74: 237-244

Frankham R (2008). Genetic adaptation to captivity in species conservation programs. Mo Ecol 17: 325-333.

Frankham R, Manning H, Margan SH, Briscoe DA (2000). Does equalization of family sizes reduce genetic adaptation to captivity? Anim Conserv 3: 357-363.

García-Dorado A, Caballero A (2000). On the average coefficient of dominance of deleterious spontaneous mutations. Genetics 155: 1991-2001.

Glémin S (2003). How are deleterious mutations purged? Drift versus nonrandom mating. Evolution 57: 2678-2687.

Hedrick PW (2001). Conservation genetics: where are we now? Trends Ecol Evol 16 629-636.

Hedrick PW, Fredrickson RJ (2008). Captive breeding and the reintroduction of Mexican and red wolves. Mol Ecol 17: 344-350.

Hellmann JJ, Pineda-Krch M (2007). Constraints and reinforcement on adaptation under climate change: selection of genetically correlated traits. Biol Conserv 137 599-609.

Ivy JA, Lacy RC (2013). A comparison of strategies for selecting breeding pairs to maximize genetic diversity retention in managed populations. J Hered 103 186-196.

Jamieson IG, Taylor SS, Tracy LN, Kokko H, Armstrong DP (2009). Why some species of birds do not avoid inbreeding: insights from New Zealand robins and saddlebacks. Behav Ecol 20: 575-584.

Jule KR, Leaver LA, Lea SEG (2008). The effects of captive experience on reintroduction survival in carnivores: a review and analysis. Biol Conserv 141 355-363.

Keller LF, Biebach I, Ewing SR, Hoeck PEA (2012). The genetics of reintroductions: inbreeding and genetic drift. In: Ewen JG, Armstrong DP, Parker KA, Seddon PJ (eds) Reintroduction Biology. Wiley-Blackwell: Oxford, UK, pp 360-394.

Kimura M, Crow JF (1963). On the maximum avoidance of inbreeding. Genet Res 4 399-415.

Kimura M, Maruyama T (1966). The mutational load with epistatic gene interactions in fitness. Genetics 54: 1337-1351.

Kokko H, Ots I (2006). When not to avoid inbreeding. Evolution 60: 467-475.

Kuhn HW (1955). The hungarian method for the assignment problem. Nav Res Logist Q 2 83-97.

ande R, Arnold SJ (1983). The measurement of selection on correlated characters. Evolution 37: 1210-1226.

Loebel DA, Nurthen RK, Frankham R, Briscoe DA, Craven D (1992). Modeling problems in conservation genetics using captive Drosophila populations-consequences of equalizing founder representation. Zoo Biol 11: 319-332.

Lynch M, Blanchard J, Houle D, Kibota T, Schultz S, Vassilieva L et al. (1999). Perspective: spontaneous deleterious mutation. Evolution 53: 645-663.

Meffert LM, Mukana N, Hicks SK, Day SB (2005). Testing alternative captive breeding strategies with the subsequent release into the wild. Zoo Biol 24 375-392.

Montgomery ME, Ballou JD, Nurthen RK, England PR, Briscoe DA, Frankham R (1997). Minimizing kinship in captive breeding programs. Zoo Biol 16: 377-389.

Mukai T, Chigusa SI, Mettler LE, Crow JF (1972). Mutation rate and dominance of genes affecting viability in Drosophila Melanogaster. Genetics 72: 335-355.

Nagylaki T (1992). Introduction to Theoretical Population Genetics. Springer-Verlag: Berlin, Germany.

O'Grady JJ, Brook BW, Reed DH, Ballou JD, Tonkyn DW, Frankham R (2006). Realistic levels of inbreeding depression strongly affect extinction risk in wild populations. Biol Conserv 133: 42-51.

Pavlidis P, Metzler D, Stephan W (2012). Selective sweeps in multilocus models of quantitative traits. Genetics 192: 225-U575.

Princée F (1995). Overcoming the constraints of social structure and incomplete pedigree data through low intensity genetic management. In: Ballou JD, Gilpin M, Foose TJ (eds) Population Management for Survival and Recovery. Columbia University Press: New York, USA, pp 124-154.

Pusey A, Wolf M (1996). Inbreeding avoidance in animals. Trends Ecol Evol 11: 201-206.

Ralls K, Ballou JD, Rideout BA, Frankham R (2000). Genetic management of chondrodystrophy in California condors. Anim Conserv 3: 145-153.

Ralls K, Ballou JD, Templeton A (1988). Estimates of lethal equivalents and the cost of inbreeding in mammals. Conserv Biol 2: 185-193.

Roze D, Blanckaert A (2014). Epistasis, pleiotropy, and the mutation load in sexual and asexual populations. Evolution 68: 137-149.

Senner J (1980). Inbreeding depression and the survival of zoo populations. In: Soulé ME, Wilcox BA (eds) Conservation Biology: An Evolutionary-Ecological Perspective. Sinaue Associates Inc.: Sunderland MA, USA, pp 151-169. 
Snyder NFR, Derrickson SR, Beissinger SR, Wiley JW, Smith TB, Toone WD et al. (1996). Limitations of captive breeding in endangered species recovery. Conserv Biol 10: 338-348.

Szulkin M, Stopher KV, Pemberton JM, Reid JM (2013). Inbreeding avoidance, tolerance, or preference in animals? Trends Ecol Evol 28: 205-211.

Theodorou K, Couvet D (2003). Familial versus mass selection in small populations. Genet Select Evol 35: 425-444.

Theodorou K, Couvet D (2004). Introduction of captive breeders to the wild: Harmful or beneficial? Conservation Genetics 5: 1-12.

Theodorou K, Couvet D (2010). Genetic management of captive populations: the advantages of circular mating. Conserv Genet 11: 2289-2297.
Wang JL (2000). Effects of population structures and selection strategies on the purging of inbreeding depression due to deleterious mutations. Genet Res 76: 75-86.

Willensdorfer M, Burger R (2003). The two-locus model of Gaussian stabilizing selection. Theor Popul Biol 64: 101-117.

Williams SE, Hoffman EA (2009). Minimizing genetic adaptation in captive breeding programs: A review. Biol Conserv 142: 2388-2400.

Wisely SM, McDonald DB, Buskirk SW (2003). Evaluation of the genetic management of the endangered black-footed ferret (Mustela nigripes). Zoo Biol 22: 287-298.

Woodworth LM, Montgomery ME, Briscoe DA, Frankham R (2002). Rapid genetic deterioration in captive populations: causes and conservation implications. Conserv Genet 3: 277-288. 